\title{
Transmission de l'Onchocerque bovine, Onchocerca gutturosa, par Culicoides
}

\author{
par O. BAIN * \\ * Laboratoire de Zoologie (Vers), associé au C.N.R.S., Muséum national d'Histoire Naturelle, \\ 43. rue Cuvier, 75231 Paris Cedex 05
}

\section{Résumé.}

Deux faits nous font penser qu'O. gutturosa est transmise non par Simulie, comme il est admis, mais par Culicoides: 1. S. ornatum, vecteur incriminé dans la transmission d'O. gutturosa, pique électivement les Bovins dans la région ventrale, mais ce sont les microfilaires d'O. lienalis qui ont cette localisation préférentielle et non celles de gutturosa qui, au contraire, sont situées dans la région dorsale. 2. La présence d'O. gutturosa dans des pays pratiquement sans Simulies.

Le cycle d'O. gutturosa chez $C$. nubeculosus s'effectue expérimentalement avec de bons taux d'infestation.

Nous pensons donc que O. gutturosa est transmise par des Culicoides et que le cycle chez Simulie, attribué à cette espèce, concerne en réalité $O$. lienalis.

\section{Summary.}

\section{Transmission of bovine Onchocerca, $O$. gutturosa, by Culicoides.}

Observations made by the author lead to suspect that $O$. gutturosa is not transmitted by Simulia but by Culicoides : 1. S. ornatum, the vector said to transmit O. gutturosa, bites preferentially the cattle in the ventral area, but the microfilariae observed in this area pertain to $O$. lienalis and not $O$. gutturosa, of which the microfilariae are, at the opposite, located in the dorsal area. 2. Records of $O$. gutturosa in places where simulia are absent, or nearly so, are in accordance with these observations.

Experimentally, the cycle of $O$. gutturosa is obtained in $C$. nubeculosus with a high level of infestation.

Thus the author concludes that $O$. gutturosa is transmitted by Culicoides and that the cycle, in Simulia, attributed to O. gutturosa, pertains in fact to O. lienalis.

Accepté le 7 juin 1979. 


\section{Conditions de l'expérience}

L'animal utilisé est un taureau importé d'Espagne (1) (Guadalquivir) et abattu à Arles, dont la densité en microfilaires dans la peau du cou est très élevée.

Les microfilaires sont extraites des «snips» du cou dans du sérum de veau. Le diagnostic spécifique est effectué sur un prélèvement d'une cinquantaine de microfilaires : ce sont toutes des microfilaires d'O. gutturosa.

Les Culicoides nubeculosus, élevés au laboratoire, sont gorgés sur la suspension de microfilaires, à travers une membrane (Parafilm). Ils sont ensuite maintenus dans une étuve à $27^{\circ} \mathrm{C}$.

Six jours et demi après le repas de sang, les Culicoides survivants sont disséqués (une quinzaine sur un lot initial d'une centaine de femelles gorgées). Un trentaine de larves sont récoltées; ce sont presque toutes des formes infestantes; il y a également quelques stades II âgés ; un Culicoides sur deux est parasité et peut contenir jusqu'à six larves infestantes dans la tête.

\section{Description du stade infestant d' O. gutturosa; comparaison avec celui d' $O$. volvulus et d' $O$. cervicalis}

La morphologie du stade infestant est indiquée sur la figure 1. La tête porte quatre petites papilles labiales et quatre papilles céphaliques plus saillantes; la capsule buccale est haute de $4-5 \mu \mathrm{m}$ et à peine cuticularisée; la queue est droite ou légèrement incurvée vers la face ventrale; elle porte un mucron terminal axial et deux mucrons sublatéraux. L'ébauche génitale est près du début de l'œsophage glandulaire chez la femelle, et près du début de l'intestin chez la larve mâle.

\section{DimENSIONS.}

Quatre larves infestantes mesurées à frais, après immobilisation en extension par la chaleur, donnent respectivement les mensurations suivantes: corps long de $860,900,820$ et $770 \mu \mathrm{m}$, large de $17,18,19$ et $19 \mu \mathrm{m}$; anneau nerveux à $94,84,84$, et $83 \mu \mathrm{m}$ de l'apex; début de la cellule excrétrice à 134, 124, 118 et $120 \mu \mathrm{m}$ de l'apex ; œsophage musculaire long de $150,155,140$ et $130 \mu \mathrm{m}$ et œsophage glandulaire long de $410,417,330$ et $355 \mu$; rectum long de 52, 62, 45 et $56 \mu \mathrm{m}$; queue longue de $47,42,38$ et $41 \mu \mathrm{m}$.

D'autres larves, également immobilisées à la chaleur, mais ensuite conservées dans l'alcool ou dans le formol (6 larves sont retrouvées), donnent des dimensions comparables.

(1) Les Bovins de nos régions ont des densités microfilariennes trop faibles pour permettre la réalisation d'un cycle expérimental. 


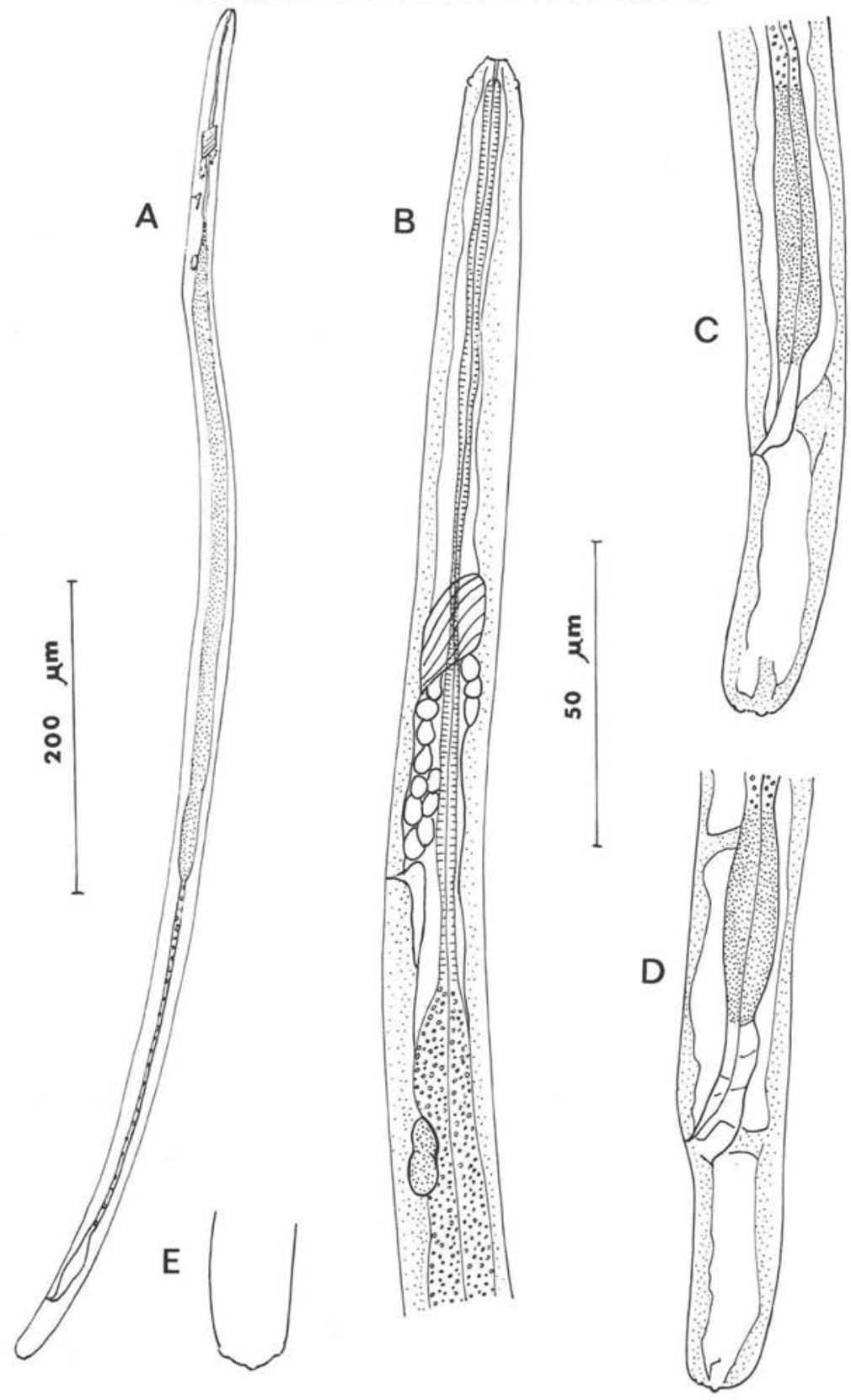

Fig. 1. Larve infestante d'O. gutturosa; A : vue latérale; B : région antérieure d'une larve vue latérale; $\mathrm{C}$ et $\mathrm{D}$ : deux régions caudales, vue latérale; $\mathrm{E}$ : extrémité caudale, vue ventrale (A, éch. $200 \mu \mathrm{m} ; \mathrm{B}, \mathrm{C}, \mathrm{D}, \mathrm{E}$, éch. $50 \mu \mathrm{m}$ ) 
Pour l'ensemble des larves infestantes, les mensurations extrêmes sont les suivantes, respectivement pour le corps, l'œsophage, l'œsophage musculaire et la queue : 680 et $900 \mu \mathrm{m} ; 360$ et $572 \mu \mathrm{m} ; 136$ et $158 \mu \mathrm{m} ; 30$ et $47 \mu \mathrm{m}$ : le rapport de la longueur de l'œsophage à celle du corps varie de 0,42 à 0,65.

Aucune particularité morphologique d'ordre qualitatif n'oppose les larves infestantes des trois espèces $O$. gutturosa, $O$. cervicalis et $O$. volvulus.

Les dimensions dont nous disposons actuellement pourraient indiquer que la longueur du corps est généralement plus faible chez $O$. volvulus et que le rapport $\frac{\text { œsophage total }}{\text { longueur du corps }}$ est plus élevé chez O. cervicalis. (Dans la note Bain et Petit, 1978, il faut lire œsophage total et non œsophage glandulaire pour les dimensions d'O. volvulus indiquées : 360 et $510 \mu \mathrm{m})$ :

Longueur moyenne du corps :

O. volvulus $725,6 \mu \mathrm{m} \pm 10,9(600$ à $825 \mu \mathrm{m}) 25$ données,

O. gutturosa $825,5 \mu \mathrm{m} \pm 20,7(680$ à $900 \mu \mathrm{m}) 10$ données,

O. cervicalis $810,9 \mu \mathrm{m} \pm 20,3(680$ à $870 \mu \mathrm{m}) 11$ données.

Moyenne du rapport : $\frac{\text { œsophage total }}{\text { longueur du corps }}$

O. volvulus $0,63 \pm 0,01(0,51$ à 0,71$) 25$ données,

O. gutturosa $0,55 \pm 0,03(0,42$ à 0,65$) 9$ données,

O. cervicalis $0,71 \pm 0,01(0,69$ à 0,73$) 5$ données.

\section{Discussion}

Tous les traités de parasitologie se fondent sur les travaux de Steward (1937), confirmés par d'autres auteurs (Gnedina, 1950 ; Supperer, 1952 ; Mikhailyuk, 1967 ; Eichler, 1971) pour indiquer que Onchocerca gutturosa est transmise par des Simulies, et plus précisément en Europe occidentale Simulium (Odagmia) ornatum Mg.

Une cause d'erreur vient du fait qu'O. gutturosa a été fréquemment confondu avec O. lienalis. Depuis l'isolement d'O. lienalis par Stiles (1892), divers auteurs (Steward, 1937 ; Caballero, 1945), dont l'argumentation a été reprise par Eichler (1973), ont conclu à l'identité des deux espèces.

D'autres auteurs, au contraire (Johnston, 1921 ; Beaver et coll., 1974 ; Eberhard, 1976), estiment qu'il s'agit de deux bonnes espèces. Nous avons récemment indiqué comme principaux caractères distinctifs le tégument de la femelle (chez gutturosa quatre stries par intercôte, ailes latérales et forte dissymétrie dorsoventrale de la musculature ; chez lienalis deux stries par intercôte; pas d'ailes ni de dissymétrie), et les microfilaires (corps mince - 3,7 à $4 \mu \mathrm{m}$ - et atténué dans la région antérieure chez gutturosa; région antérieure large comme la partie moyenne du corps - 5 à $5,3 \mu \mathrm{m}-$ chez lienalis) (Bain et coll., 1978).

Ainsi qu'il est indiqué plus haut, les localisations privilégiées des deux espèces de microfilaires sont distinctes: $O$. gutturosa prédomine dans le derme du cou et du 
dos (Le Roux, 1947 ; Fain et coll., 1955 ; Bain et coll., 1977) ; O. lienalis, au contraire, se trouve presque exclusivement dans la peau du ventre (Bain et coll., 1978).

La transmission d'O. gutturosa par $S$. (O.) ornatum qui, en Europe occidentale, pique presque exclusivement le ventre des Bovins (Davies, 1957 ; Eichler, 1971), paraît donc improbable.

Par ailleurs, l'examen de Bovins africains provenant de régions du Sahel apparemment dépourvus de Simulies (fide Le Berre) montrent des infestations constantes et très riches à $O$. gutturosa.

Nous soupçonnions donc, à la suite de Le Roux (1947), qu'O. gutturosa était transmise par Culicoides et non par Simulie (Bain et coll., 1978).

L'expérience a été difficile à réaliser, car les Bovins sacrifiés dans les abattoirs en France n'ont que des infections rares et faibles, et, en Afrique occidentale, nous ne disposions pas de bons élevages de Culicoides.

Le premier essai expérimental réalisé dans de bonnes conditions grâce à un taureau provenant du Guadalquivir ayant donné un résultat massivement positif, nous n'hésitons pas à conclure qu'O. gutturosa est transmise dans la nature par des Culicoides et que les expériences de transmission par Simulies, qui avaient été rapportées précédemment, concernent presque certainement $O$. lienalis.

\section{Bibliographie}

Bain O., Denke A.-M., Amégée Y., Chabaud A.-G. (1977): Les Onchocerques des Bovins au Togo; les microfilaires et leurs distributions. Ann. Univ. Bénin, Togo, 3, 117-123.

Bain O., Petit G. (1978) : Redescription du stade infestant d'Onchocerca cervicalis R. et H., 1910. Ann. Parasit. hum. comp., 53, 315-318.

Bain O., Petit G., Poulain B. (1978): Validité des deux espèces Onchocerca lienalis et O. gutturosa chez les Bovins. Ann. Parasit. hum. comp., 53, 421-430.

Beaver P. C., Horner G.S., Bilos J.Z. (1974) : Zoonotic onchocercosis in a resident of Illinois and observations on the identification of Onchocerca species. Amer. J. Trop. Med. Hyg., 23, 595607.

Caballero C.E. (1945): Estudios helmintologicos de la région oncocercosa de Mexico y de la Republica de Guatemala. Nematoda. 3rd parte. Filarioidea II. El genero Onchocerca. An. Inst. Biol., 16, 367-409.

Davies L. (1957): A study of the blackfly, Simulium ornatum Mg (Diptera), with particular reference to its activity on grazing cattle. Bull. ent. Res., 48, 407-424.

Eberhard M. L. (1976): The morphology of Onchocerca species (Nematoda: Filarioidea) found in cattle in the United States. 22 juin 1976, 62 p. polycopiées, dissertation, Tulane University.

Eichler D. A. (1971): Studies on Onchocerca gutturosa (Neumann, 1910) and its development in Simulium ornatum (Meigen, 1818). II Behaviour of $S$. ornatum in relation to the transmission of O. gutturosa. J. Helm., 45, 259-270.

Eichler D. A., Nelson G.S. (1971): idem. I. Oservations on O. gutturosa in cattle in South East England. J. Helm., 45, 245-258.

Eichler D. A. (1973): idem. IV. Systematics of O. gutturosa. J. Helm., 47, 89-96.

Fain A., Hérin V., Thienpont D. (1955): Filarioses des bovidés au Ruandi-Urundi. III. Etude parasitologique. B) Filaires des genres Setaria et Onchocerca et microfilaires sanguines et dermiques. Ann. Soc. Belge Med. Trop., 35, 555-582.

Gnédina M.D. (1950): Biologie du nématode Onchocerca gutturosa, Neumann (1910), parasite du bétail. Dokl. Akad. Nauk. S.S.S.R., 70, 169-171 (en russe). 
Johnston T.H. (1921): Onchocerciasis of Queensland cattle. Trans. Proc. R. Soc. South Australia, 45, 231-247.

Le Roux P. L. (1947): Demonstration: 7. Proximal part of the M. biceps brachii from an ox showing Onchocerca gutturosa infestation, Mazabuka, Northern Rhodesia. Trans. R. Soc. Trop. Med. Hyg., 41, 9.

Mikhailyuk A.P. (1967): O. gutturosa et O. lienalis dans la zone de steppe boisée d'Ukraine. Veterinariya, Kiev, 11, 62-67 (en russe).

Steward J.S. (1937): The occurrence of Onchocerca gutturosa, Neumann in cattle in England, with an account of its life history and development in Simulium ornatum Mg. Parasitology, 29, 212-219.

Stiles H.W. (1892): A checklist of animal parasites of cattle, with a request to veterinarians and zoologists. J. Comp. med. vet. Arch., 13, 346-350.

Supperer R. (1952): Ueber das Vorkommen der Filarie (s.l.) Onchocerca gutturosa, Neumann in Rindern in Osterreich und ihre Entwicklung in der Kriebelmüche Odagmia ornata Mg. Wiener Tierärzt. Monastsoch., 39, 173-179.

Tous droits de traduction, d'adaptation et de reproduction par tous procédés pour tous pays

La loi du 11 mars 1957 n'autorisant, aux termes des alinéas 2 et 3 de l'article 41, d'une part, que les «copies ou reproductions strictement réservées à l'usage privé du copiste et non destinées à une utilisation collective $\gg$ et, d'autre part, que les analyses et les courtes citations dans un but d'exemple et d'illustration, «toute représentation ou reproduction intégrale ou partielle, faite sans le consentement de l'auteur ou de ses ayants-droit ou ayants-cause, est illicite 》 (alinéa $1^{\text {or }}$ de l'article 40).

Cette représentation ou reproduction, par quelque procédé que ce soit, constituerait donc une contrefaçon sanctionnée par les articles 425 et suivants du Code pénal.

Masson, éditeur, Paris. - Dépôt légal : 1979. - $\mathrm{N}^{\circ}$ d’ordre : 5171. - $4^{\circ}$ trimestre 1979 